\title{
30 parameter version of the MSSM in light of the $B_{s} \rightarrow \mu^{+} \mu^{-}$observation
}

\author{
S. S. AbdusSalam ${ }^{1, *}$ and L. Velasco-Sevilla ${ }^{2, \dagger}$ \\ ${ }^{1}$ Department of Physics, Shahid Beheshti University, Tehran 19839, Islamic Republic of Iran \\ ${ }^{2}$ University of Bergen, Department of Physics and Technology, P.O. Box 7803, 5020 Bergen, Norway
}

(Received 16 August 2017; published 7 March 2018)

\begin{abstract}
The R-parity conserving minimal supersymmetric standard model (MSSM) in light of the decay $B_{s} \rightarrow \mu^{+} \mu^{-}$with near-standard model branching ratio is an interesting platform for studying the complementarity between direct and indirect searches for beyond the standard model physics. Based on this, we have analysed the possible impact of the $B_{s} \rightarrow \mu^{+} \mu^{-}$observation on the posterior sample from the global fit of a 30-parameter MSSM (MSSM-30), and the related Wilson coefficients. The MSSM-30 is a systematically constructed, symmetry-guided, MSSM parameterisation, as opposed to the traditional frames (e.g., pMSSM) with crude treatment of flavor violation parameters. This paper illustrates why phenomenological frames like the MSSM-30 should be preferred to study flavor physics. For the current and future B-physics experimental precision, such a consideration is crucial for suitably assessing supersymmetric contributions to flavor observables.
\end{abstract}

DOI: 10.1103/PhysRevD.97.055010

\section{INTRODUCTION}

The decay $B_{s} \rightarrow \mu^{+} \mu^{-}$has been used traditionally as an indicator of how contributions from extended Higgs sectors, with respect to the standard model (SM), can give sizeable contributions to leptonic decays. This happens because its branching fraction undergoes a helicity suppression by $m_{\mu}^{2} / M_{B_{s}}^{2}$, where $m_{\mu}$ is the mass of the muon and $M_{B_{s}}$ is the mass of the $B_{s}$ meson. This helicity suppression can be lifted in models with extra Higgs doublets where chirality-changing quark flavor violation is present and it is particularly strong for large values of $\tan \beta[1,2]$. The chirality-changing quark flavor violation contributions to $B_{s} \rightarrow \mu^{+} \mu^{-}$are proportional to $m_{B_{s}}^{2} / m_{b}^{2}$ instead. Hence, these decays provide a good opportunity to look for physics beyond the standard model (BSM). For the particular BSM case of the R-parity conserving minimal supersymmetric standard model (MSSM), with diagonal soft-squared mass matrices and trilinear terms, analytical approximations indicate that the $B_{s} \rightarrow \mu^{+} \mu^{-}$decay amplitude can be proportional to $\tan ^{3} \beta$ [3-7]. Here $\tan \beta$ is the ratio of the MSSM Higgs fields vacuum expectation values: $\left\langle H_{2}\right\rangle /\left\langle H_{1}\right\rangle$ that can take values between 2 and 60. However, full-fledged (numerical) analyses including global fits of models to

\footnotetext{
*abdussalam@sbu.ac.ir

Liliana.Velasco-Sevilla@ift.uib.no
}

Published by the American Physical Society under the terms of the Creative Commons Attribution 4.0 International license. Further distribution of this work must maintain attribution to the author(s) and the published article's title, journal citation, and DOI. Funded by SCOAP. experimental data have shown that BSM contributions to $B_{s} \rightarrow \mu^{+} \mu^{-}$behave in a multidimensional manner.

Experimental constraints mostly suppress, or require opposite signs with similar magnitudes, the various supersymmetric contributions with respect to the SM one. Examples showing the manifestation of this suppression were shown within the phenomenological MSSM (pMSSM) framework [8,9], where the $\operatorname{BR}\left(B_{s} \rightarrow \mu^{+} \mu^{-}\right)$ posterior distribution lies around the SM prediction despite the moderately high values of $\tan \beta$, and in the more recent work of [10], as required by experiments which indicate the absence of large deviations of $\operatorname{BR}\left(B_{s} \rightarrow \mu^{+} \mu^{-}\right)$from the SM prediction [11-18].

Given the amazing consistency of the predictions of the SM with flavor observables one may wonder if in supersymmetry there is a mechanism, that just as it happens in the SM, effectively forbids flavor changing neutral current (FCNC) processes and controls $C P$ violation. Specific models with a MSSM spectrum which avoid FCNC and $C P$ violating processes can be constructed successfully [19-21]. However, without a specific model for generation of flavor within the MSSM, it is necessary to work within a phenomenological framework with a systematically constructed parametrisation of flavor violation. Following this rationale, we consider a MSSM framework with 30 parameters [22,23] which goes beyond the constrained MSSM (see [24-30] for related works within the constrained MSSM set ups) and the pMSSM [8,31,32] where flavor violation in the SUSY breaking mass terms is manipulated by hand albeit with reasonable motivations.

In the pMSSM there is no information about how flavor violation in the soft-squared masses and trilinear terms 
generated by radiative corrections will impact flavor observables. We know that once full $3 \times 3$ Yukawa matrices are considered, flavor violation is automatically generated through radiative effects. In the MSSM-30, a counting rule keeps track of the hierarchical structure of the Yukawa matrices, which are expanded in terms of the Cabibbo angle. Then trilinear terms and soft squared masses can be expanded in that basis. The off-diagonal parameters generated in that way can be thought of as the effective off-diagonal parameters generated through radiative corrections. This rationale discards dangerous terms for $\mathrm{FCNC}$ and $C P$ violation. In this sense, this work builds further on the project for MSSM explorations within systematically built frames, in this case a specific frame for flavor violation, deriving inference from experimental data $[8,23,32-39]$ with fewer theoretical or traditional prejudices compared to other MSSM phenomenology frameworks.

The structure of flavor violation ${ }^{1}$ and the flat distribution chosen for this work make our results obviously model dependent but one of our points is to exemplify how a realistic treatment for flavor effects in the MSSM can influence the allowed parameter space regions of supersymmetric parameters. The flavor structure that we have chosen is a realization of minimal flavor violation (MFV), and within this framework the posterior sample from the MSSM-30 fit in [23] indicates results which are more restrictive than those from the LHC searches for gluinos and squarks. The MFV parametrization favours heavy gluinos and squarks [23], in order to satisfy flavor and electric dipole moment constraints.

This work is organised as follows. In Sec. II, we present a brief review of the MSSM-30 construction and the global fit of its parameters to data from indirect searches for BSM physics. The effect of the $B_{s} \rightarrow \mu^{+} \mu^{-}$measurement, and the possible future accuracy of the measurement, on the MSSM-30 parameters is analysed there. In Sec. III, we present respectively the numerical anatomy and analyses of the Wilson coefficients, in terms of contributions to $B_{s} \rightarrow$ $\mu^{+} \mu^{-}$classified according to kind of diagrams and kind of particles. For some interesting points, we give comparisons and contrast the MSSM-30 results to the pMSSM case. The summary and conclusions are presented in Sec. IV.

\section{THE MSSM-30 PARAMETERS IN LIGHT OF THE $B_{s} \rightarrow \mu^{+} \mu^{-}$OBSERVATION}

Here we briefly set the context of our analyses. First, the 30-parameter-MSSM framework is presented and contrasted with the pMSSM giving emphasis to the constraints on the two parameters most sensitive to the $\operatorname{BR}\left(B_{s} \rightarrow \mu^{+} \mu^{-}\right)$ observable: $\tan \beta$ and $m_{A}$. Second, the possible impact of future experimental precision in the $\operatorname{BR}\left(B_{s} \rightarrow \mu^{+} \mu^{-}\right)$

\footnotetext{
${ }^{1}$ The way flavor is set up at in [22] takes $Y_{u}=\lambda_{u} V$ and $Y_{d}$ diagonal, where $\lambda_{u}$ is the diagonal matrix of Yukawa couplings and $V$ is the Cabibbo-Kobayashi-Maskawa (CKM) matrix.
}

measurement is addressed. Finally, we comment on the possible impact of a future $\operatorname{BR}\left(B_{s} \rightarrow \mu^{+} \mu^{-}\right)$precision measurement on the $\operatorname{MSSM}\left(m_{A}, \tan \beta\right)$ plane.

\section{A. The MSSM-30 frame}

In [23] the minimal flavor violation MSSM parameters selection scheme leads to an MSSM frame with 30 parameters:

$$
\begin{aligned}
\underline{\theta} \equiv & \left\{\operatorname{Re}\left[\tilde{M}_{1,2}\right], M_{3}, M_{A}, \tan \beta, \operatorname{Im}\left[\tilde{M}_{1,2}, \tilde{\mu}\right],\right. \\
& a_{1,2,3,6,7}, \operatorname{Re}\left[\tilde{a}_{4,5,8}\right], \operatorname{Im}\left[\tilde{a}_{4,5,8}\right], x_{1,2}, y_{1,3,6,7}, \\
& \left.\operatorname{Re}\left[\tilde{y}_{4,5}\right], \operatorname{Im}\left[\tilde{y}_{4,5}\right]\right\},
\end{aligned}
$$

which stem from the terms

$$
\begin{aligned}
\tilde{M}_{1} & =e^{i \phi_{1}} M_{1}, \\
\tilde{M}_{2} & =e^{i \phi_{2}} M_{2}, \quad M_{3}, \\
\tilde{\mu} & =\mu e^{i \phi_{\mu},} \quad M_{A}, \tan \beta, \\
M_{Q}^{2} & =a_{1} \mathbf{1}+x_{1} X_{13}+y_{1} X_{1}, \\
X_{1} & =\operatorname{diag}\left\{0,0, \delta_{3 i} \delta_{3 j}\right\}, \\
X_{13} & =V_{3 i}^{*} V_{3 j}, \\
M_{U}^{2} & =a_{2} \mathbf{1}+x_{2} X_{1}, \\
M_{D}^{2} & =a_{3} \mathbf{1}+y_{3} X_{1}, \\
M_{L}^{2} & =a_{6} \mathbf{1}+y_{6} X_{1}, \\
M_{E}^{2} & =a_{7} \mathbf{1}+y_{7} X_{1}, \\
A_{E} & =\tilde{a}_{8} X_{1}, \\
A_{U} & =\tilde{a}_{4} X_{5}+\tilde{y}_{4} X_{1}, \\
X_{5} & =\delta_{3 i} V_{3 j} \\
A_{D} & =\tilde{a}_{5} X_{1}+\tilde{y}_{5} X_{5},
\end{aligned}
$$

Here $i$ and $j$ run over as sparticles family indices, $V$ is the SM CKM matrix, 1 the unit matrix and $\delta$ the Kronecker delta function. The gaugino mass parameters $\tilde{M}_{1}, \tilde{M}_{2}$ were allowed in the range -4 to $4 \mathrm{TeV}$ for both real and imaginary parts. The gluino mass term, $M_{3}$, is allowed in $100 \mathrm{GeV}$ to $4 \mathrm{TeV}$. The parameters $a_{1,2,3,6,7}$ were varied within the range $(100 \mathrm{GeV})^{2}$ to $(4 \mathrm{TeV})^{2}$ and $-(4 \mathrm{TeV})^{2}$ to $(4 \mathrm{TeV})^{2}$ for $x_{1,2}, y_{1,3,6,7}$. The trilinear coupling terms $\operatorname{Re}\left[\tilde{a}_{4,5,8}\right], \operatorname{Im}\left[\tilde{a}_{4,5,8}\right], \operatorname{Re}\left[\tilde{y}_{4,5}\right]$, and $\operatorname{Im}\left(\tilde{y}_{4,5}\right)$ were varied within $-8 \mathrm{TeV}$ to $8 \mathrm{TeV}$. Here $m_{A}$ was allowed in $100 \mathrm{GeV}$ to $4 \mathrm{TeV}$ while the Higgs doublets mixing term, both real and imaginary parts $(\operatorname{Re}[\tilde{\mu}], \operatorname{Im}[\tilde{\mu}])$ in the range -4 to $4 \mathrm{TeV}$. In comparison, the pMSSM parameters are

$\underline{\theta}=\left\{M_{1,2,3} ; m_{\tilde{f}_{Q, U, D, L, E}}^{\text {3rdgen }}, m_{\tilde{f}_{Q, U, D, L, E}}^{1 \mathrm{st} / 2 \text { ndgen }} ; A_{t, b, \tau, \mu=e}, m_{H_{u, d}}^{2}, \tan \beta\right\}$,

where $M_{1,2,3}$ are as for MSSM-30, $m_{\tilde{f}}$ the sfermion mass parameters were allowed in the range $100 \mathrm{GeV}$ to $4 \mathrm{TeV}$. 
The trilinear couplings $A_{t, b, \tau, \mu=e} \in[-8,8] \mathrm{TeV}$. The Higgs doublet masses $m_{H_{1}}^{2}, m_{H_{2}}^{2}$ were allowed according to $m^{2} \in \operatorname{sign}(m)[-4,4]^{2} \mathrm{TeV}^{2}$. Here $\operatorname{sign}(\mu)$ is the sign of the Higgs doublets mixing parameter (allowed to be randomly \pm 1 ). The SM parameters were fixed at their experimentally determined central values for the MSSM-30 but varied in a Gaussian manner for the pMSSM.

\section{B. The MSSM-30 global fit to data}

The posterior distribution used for our analysis came from a Bayesian fit of the MSSM-30 to data [23]. In order to make this paper a self-contained exposition of the statistical details of our work, in what follows we describe the fitting procedure.

The Bayesian fit was performed within a context, $\mathcal{H}$, where the MSSM-30 neutralino lightest supersymmetric particle is assumed to be a least part of the cold dark matter $(\mathrm{CDM})$ relic. The thirty parameters, detailed in Eq. (1), were varied according to a flat prior probability density, $p(\underline{\theta} \mid \mathcal{H})$. The SM parameters fixed were: the mass of the Z-boson, $m_{Z}=91.2 \mathrm{GeV}$, the top quark mass, $m_{t}=165.4 \mathrm{GeV}$, the bottom quark mass, $m_{b}=4.2 \mathrm{GeV}$, the electromagnetic coupling, $\alpha_{e m}^{-1}=127.9$, and the strong interaction coupling, $\alpha_{s}=0.119$.

The data set, $\underline{d}$, used for fitting the MSSM-30 are summarised in Table I. It is composed of the experimental central values, $\mu_{i}$, and errors, $\sigma_{i}$, for the Higgs boson mass, the electroweak physics, B-physics, dipole moment of leptons and the CDM relic density observables set

$$
\begin{aligned}
\underline{O} \equiv & \left\{m_{h}, m_{W}, \Gamma_{Z}, \sin ^{2} \theta_{\mathrm{eff}}^{l e p}, R_{l}^{0}, R_{b, c}^{0}, A_{F B}^{b, c}, A^{l}=A^{e}, A^{b, c},\right. \\
& \operatorname{BR}\left(B \rightarrow X_{s} \gamma\right), \operatorname{BR}\left(B_{s} \rightarrow \mu^{+} \mu^{-}\right), \Delta M_{B_{s}}, R_{\mathrm{BR}\left(B_{u} \rightarrow \tau \nu\right)}, \\
& \left.\Omega_{C D M} h^{2}, \operatorname{Br}\left(B_{d} \rightarrow \mu^{+} \mu^{-}\right), \Delta M_{B_{d}}, d_{e, \mu, \tau}\right\} .
\end{aligned}
$$

Using the data set described above, an MSSM-30 likelihood distribution $p(\underline{d} \mid \underline{\theta}, \mathcal{H})$ was constructed as

$$
p(\underline{d} \mid \underline{\theta}, \mathcal{H})=L(x) \prod_{i} \frac{\exp \left[-\left(O_{i}-\mu_{i}\right)^{2} / 2 \sigma_{i}^{2}\right]}{\sqrt{2 \pi \sigma_{i}^{2}}},
$$

where the index $i$ runs over the list of observables $\underline{O}$, the variable $x$ represents the predicted value of neutralino CDM relic density at an MSSM-30 parameter space point and

$L(x)=\left\{\begin{array}{ll}1 /\left(y+\sqrt{\pi s^{2} / 2}\right) & \text { if } x<y \\ \exp \left[-(x-y)^{2} / 2 s^{2}\right] /\left(y+\sqrt{\pi s^{2} / 2}\right) & \text { if } x \geq y\end{array}\right.$.

Here $y=0.11$ is the CDM relic density central value and $s=0.02$ the corresponding inflated (to allow for theoretical uncertainties) error.

By passing parameters to SPHENO [53,54] via the SLHA2 [55] interface, the corresponding MSSM-30 predictions for the branching ratios $\operatorname{BR}\left(B_{s} \rightarrow \mu^{+} \mu^{-}\right)$, $\mathrm{BR}(B \rightarrow s \gamma), R_{\mathrm{BR}\left(B_{u} \rightarrow \tau \nu\right)}, \operatorname{BR}\left(B_{d} \rightarrow \mu^{+} \mu^{-}\right), \Delta M_{B_{s}}, \Delta M_{B_{d}}$ and $d_{e, \mu, \tau}$ were obtained. Similarly, using the SLHA1 [56] interface, the neutralino CDM relic density was computed using MICROMEGAs [57], while SUSYPOPE [58,59] was used for computing precision observables that include the $W$-boson mass $m_{W}$, the effective leptonic mixing angle variable $\sin ^{2} \theta_{\text {eff }}^{\text {lep }}$, the total $Z$-boson decay width, $\Gamma_{Z}$, and the other electroweak observables whose experimentally determined central values and associated errors are summarised in Table I.

Using MultiNest $[60,61]$ which implements the nested sampling algorithm [62], Bayes' theorem then gives the MSSM-30 posterior probability distribution

TABLE I. The experimental results used for the Bayesian fit of the MSSM-30 parameters.

\begin{tabular}{lccc}
\hline \hline Observable & Constraint & Observable & Constraint \\
\hline$m_{W}[\mathrm{GeV}]$ & $80.399 \pm 0.023[40]$ & $A^{l}=A^{e}$ & $0.1513 \pm 0.0021[41]$ \\
$\Gamma_{Z}[\mathrm{GeV}]$ & $2.4952 \pm 0.0023[41]$ & $A^{b}$ & $0.923 \pm 0.020[41]$ \\
$\sin ^{2} \theta_{\mathrm{eff}}^{l e p}$ & $0.2324 \pm 0.0012[41]$ & $A^{c}$ & $0.670 \pm 0.027[41]$ \\
$R_{l}^{0}$ & $20.767 \pm 0.025[41]$ & $\mathrm{BR}\left(B_{s} \rightarrow \mu^{+} \mu^{-}\right)$ & $3.2_{-1.2}^{+1.5} \times 10^{-9}[16]$ \\
$R_{b}^{0}$ & $0.21629 \pm 0.00066[41]$ & $\Delta M_{B_{s}}$ & $17.77 \pm 0.12 \mathrm{ps}^{-1}[42]$ \\
$R_{c}^{0}$ & $0.1721 \pm 0.0030[41]$ & $R_{B r\left(B_{u} \rightarrow \tau \nu\right)}$ & $1.49 \pm 0.3091[43]$ \\
$A_{\mathrm{FB}}^{b}$ & $0.0992 \pm 0.0016[41]$ & $\Delta M_{B_{d}}$ & $0.507 \pm 0.005 \mathrm{ps}^{-1}[44]$ \\
$A_{\mathrm{FB}}^{c}$ & $0.0707 \pm 0.0035[41]$ & $\Omega_{\mathrm{CDM}} h^{2}$ & $0.11 \pm 0.02[45]$ \\
$m_{h}[\mathrm{GeV}]$ & $125.6 \pm 3.0[46,47]$ & $\mathrm{BR}\left(B_{d} \rightarrow \mu^{+} \mu^{-}\right)$ & $<1.8 \times 10^{-8}[48]$ \\
$d_{\mu}$ & $<2.8 \times 10^{-19}[49]$ & $\mathrm{BR}\left(B \rightarrow X_{s} \gamma\right)$ & $(3.52 \pm 0.25) \times 10^{-4}[50]$ \\
$d_{\tau}$ & $<1.1 \times 10^{-17}[51]$ & $d_{e}$ & $<1.6 \times 10^{-27}[52]$ \\
\hline \hline
\end{tabular}




$$
p(\underline{\theta} \mid \underline{d}, \mathcal{H}) \propto p(\underline{d} \mid \underline{\theta}, \mathcal{H}) \times p(\underline{\theta} \mid \mathcal{H}) .
$$

\section{C. $\operatorname{BR}\left(B_{s} \rightarrow \mu^{+} \mu^{-}\right)$prediction and measurement}

The tagged average branching fraction of the rare decay $\operatorname{BR}\left(B_{s} \rightarrow \mu^{+} \mu^{-}\right)$is given by

$$
\begin{aligned}
\operatorname{BR}\left(B_{s} \rightarrow \mu^{+} \mu^{-}\right) \\
=\frac{G_{F}^{2} \alpha^{2}}{64 \pi^{3}} f_{B_{s}}^{2} m_{B_{s}}^{3}\left|V_{t b} V_{t s}^{*}\right|^{2} \tau_{B_{s}} \sqrt{1-\frac{4 m_{\mu}^{2}}{m_{B_{s}}^{2}}} \\
\quad \times\left[\left(1-\frac{4 m_{\mu}^{2}}{m_{B_{s}}^{2}}\right) \frac{m_{B_{s}}^{2}}{m_{b}^{2}}\left|C_{S}-C_{S}^{\prime}\right|^{2}\right. \\
\left.+\left|\frac{m_{B_{s}}}{m_{b}}\left(C_{P}-C_{P}^{\prime}\right)+2\left(C_{10}-C_{10}^{\prime}\right) \frac{m_{\mu}}{m_{B_{s}}}\right|^{2}\right],
\end{aligned}
$$

where the operators that we use above are related to those of [63] by

$$
\begin{aligned}
C_{S}=X\left(C_{L L}^{S}+C_{L R}^{S}\right), & C_{S}^{\prime}=X\left(C_{R R}^{S}+C_{R L}^{S}\right), \\
C_{P}=X\left(-C_{L L}^{S}+C_{L R}^{S}\right), & C_{P}^{\prime}=X\left(C_{R R}^{S}-C_{R L}^{S}\right), \\
C_{10}=X\left(-C_{L L}^{V}+C_{L R}^{V}\right), & C_{10}^{\prime}=X\left(C_{R R}^{V}-C_{R L}^{V}\right),
\end{aligned}
$$

for $X=\pi /\left(\sqrt{2} G_{F} \alpha V_{t s}^{*} V_{t b}\right)$, and the Wilson operators and coefficients are defined through the Hamiltonian as

$$
\begin{aligned}
\mathcal{H} & =-\sum_{X, Y} O_{X Y}^{V} C_{X Y}^{V}+O_{X Y}^{S} C_{X Y}^{S}, \\
O_{X Y}^{V} & =\left(d_{J} \gamma_{\mu} P_{X} d_{I}\right)\left(\ell_{B} \gamma^{\mu} P_{Y} \lambda_{A}\right), \\
O_{X Y}^{S} & =\left(d_{J} P_{X} d_{I}\right)\left(\ell_{B} P_{Y} \lambda_{A}\right), \\
X, Y & =L, R
\end{aligned}
$$

where $O^{V}$ are vector and $O^{S}$ scalar operators respectively and $P_{X}$ are the chirality projectors. The contributions proportional to $\left|C_{S}-C_{S}^{\prime}\right|^{2}$ and $\left|C_{P}-C_{P}^{\prime}\right|^{2}$ are not any longer proportional to $m_{\mu}^{2} / m_{B_{s}}^{2}$ and hence lift the helicity suppression exhibited in the SM. We use SUSY_FLAVOR [64] to obtain the contributions from the different particles and kinds of diagrams, ${ }^{2}$ Higgs and $\mathrm{Z}$ penguins and box diagrams.

It is well established that in the SM, $C_{10}$ gets its larger contribution from the $Z$ penguin with a top loop, about $75 \%$ and its second largest contribution from the $W$ box, $24 \%$ and we have

$$
\mathrm{BR}\left(B_{s} \rightarrow \mu^{+} \mu^{-}\right)^{\mathrm{SM}}=(3.25 \pm 0.17) \times 10^{-9} .
$$

\footnotetext{
${ }^{2}$ For this case, we use a modified version of the program, for which we have explicitly checked those contributions with the help of Refs. [1,2,6,63,65,66].
}

The experimental measured quantity (denoted here with an overline) is the untagged branching fraction which is related to Eq. (8), the theoretical (tagged) expression, as

$\mathrm{BR}\left(B_{s} \rightarrow \mu^{+} \mu^{-}\right)=\left[\frac{1-y_{s}^{2}}{1+\mathcal{A}_{\Delta \Gamma}^{\mu \mu} y_{s}}\right] \overline{\mathrm{BR}}\left(B_{s} \rightarrow \mu^{+} \mu^{-}\right)$,

where $y_{s}=\Delta \Gamma_{s} / 2 \Gamma_{s}, \Delta \Gamma_{s}$ being the decay width difference between the $B_{s}$ mass eigenstates and $\Gamma_{s}=\tau_{B_{s}}^{-1}$ is the average $B_{s}$ decay width, using the LHCb measurement $\left(y_{s}=0.087 \pm 0.014\right.$ [67]), and that in the $\mathrm{SM} \mathcal{A}_{\Delta \Gamma}^{\mu \mu}=1$, we obtain $^{3}$

$$
\overline{\mathrm{BR}}\left(B_{s} \rightarrow \mu^{+} \mu^{-}\right)^{\mathrm{SM}}=(3.56 \pm 0.18) \times 10^{-9},
$$

on the other hand, the experimental value measured by the LHCb Collaboration is [18,70], including Run 1 and Run 2 data,

$$
\overline{\mathrm{BR}}\left(B_{s} \rightarrow \mu^{+} \mu^{-}\right)=\left(3.0 \pm 0.6_{-0.2}^{+0.3}\right) \times 10^{-9} .
$$

while the CMS value is [71],

$$
\overline{\operatorname{BR}}\left(B_{s} \rightarrow \mu^{+} \mu^{-}\right)=\left(2.8 \pm 0.5_{-0.2}^{+0.3}\right) \times 10^{-9} .
$$

As we can see, both values in agreement with the SM. In the MSSM, $\mathcal{A}_{\Delta \Gamma}^{\mu \mu}=\left(|P|^{2} \cos \left(2 \varphi_{P}\right)-|S|^{2} \cos \left(2 \varphi_{S}\right)\right)$ / $\left(|P|^{2}+|S|^{2}\right), \quad[68]$, where $\varphi_{S}=\arg (S), \quad \varphi_{P}=\arg (P)$, and $S$ and $P$ are related to our notation for the Wilson coefficients as follows

$$
\begin{gathered}
S=\sqrt{1-4 \frac{m_{\mu}^{2}}{m_{B_{s}}^{2}} \frac{m_{B_{s}}^{2}}{2 m_{\mu}} \frac{1}{m_{b}+m_{s}} \frac{C_{S}-C_{S}^{\prime}}{C_{10}^{\mathrm{SM}}} \frac{m_{B_{s}}}{m_{b}},} \\
P=\frac{C_{10}}{C_{10}^{\mathrm{SM}}}+\frac{m_{B_{s}}^{2}}{2 m_{\mu}} \frac{1}{m_{b}+m_{s}} \frac{C_{P}-C_{P}^{\prime}}{C_{10}^{\mathrm{SM}}} \frac{m_{B_{s}}}{m_{b}} .
\end{gathered}
$$

In the upper panels of Fig. 1 we compare the planes $\tan \beta$ vs the tagged value of $\operatorname{BR}\left(B_{s} \rightarrow \mu^{+} \mu^{-}\right)$(top-left), as produced by the official 2 253 version of SUSY FLAVOR, and $\tan \beta$ vs the untagged value of $\operatorname{BR}\left(B_{s} \rightarrow\right.$ $\mu^{+} \mu^{-}$) (top-right) using a modified version of it. This comparison shows the importance of appropriately comparing the measurement of the $\operatorname{BR}\left(B_{s} \rightarrow \mu^{+} \mu^{-}\right)$with the theoretical value. Although the contributions from the prefactors in Eq. (12) do not differ greatly from point to point (due to the smallness of the supersymmetric contributions), they have a significant impact in pushing up the values of $\operatorname{BR}\left(B_{s} \rightarrow \mu^{+} \mu^{-}\right)$. In the lower part of the figure we present just the tagged distribution for the linear prior of

\footnotetext{
${ }^{3}$ We note that this value is in agreement with [68], but a better treatment of $\mathrm{NLO} \mathrm{EW}$ corrections to $C_{10}$ place $\overline{\mathrm{BR}}\left(B_{s} \rightarrow\right.$ $\left.\mu^{+} \mu^{-}\right)^{\mathrm{SM}}=(3.65 \pm 0.23) \times 10^{-9}$ [69].
} 

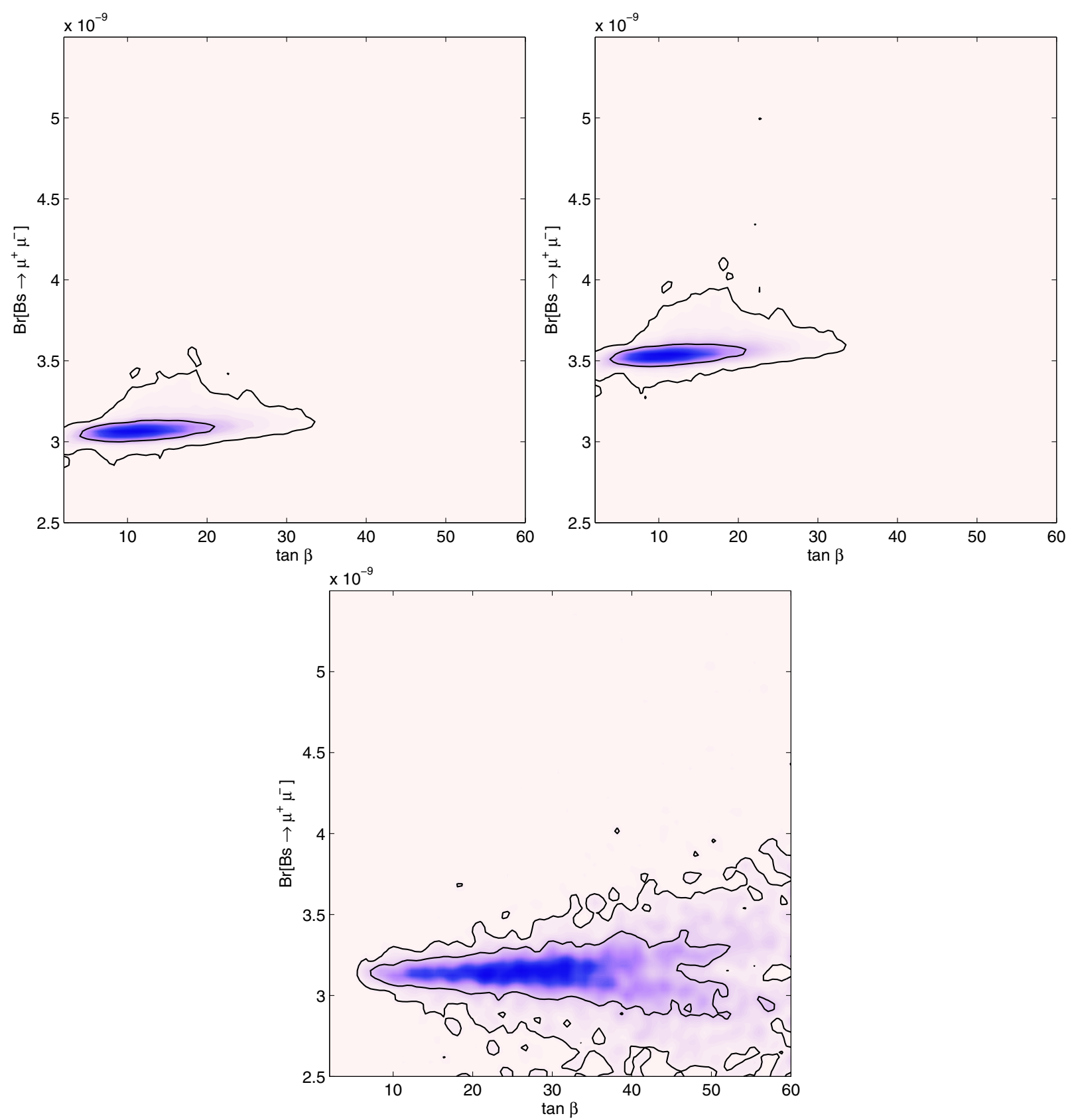

FIG. 1. $\tan \beta$ vs $\operatorname{BR}\left(B_{s} \rightarrow \mu^{+} \mu^{-}\right)$for the MSSM-30 sample (top) and for the pMSSM (bottom). For the MSSM-30 sample we have plotted (left) the tagged contribution as produced by the official 2_53 version of SUSY_FLAVOR and the untagged values (right) using a modified version of it. One of the main results of our work is to note that whereas values for $\tan \beta<10$ are excluded in the pMSSM, for the MSSM-30 such low values of $\tan \beta$ are possible.

the pMSSM, which is in agreement with that of [9]. In the plane $\tan \beta \operatorname{vs~} \operatorname{BR}\left(B_{s} \rightarrow \mu^{+} \mu^{-}\right)$is clear that for the MSSM30 , contrary to the pMSSM, values of $\tan \beta<10$ are not excluded. This shows that allowing a richer structure in the soft-squared terms, opens up regions of parameter space in comparison to the pMSSM. One of the main results of this work is that we found that the MSSM-30 predictions for $\operatorname{BR}\left(B_{s} \rightarrow \mu^{+} \mu^{-}\right)$with $\tan \beta \in(10,20)$ are within the experimental limit, while for the pMSSM in that range are not. For the pMSSM instead the preferred values for $\tan \beta$ are above 25 .

From the second plot of Fig. 1, we can see that supersymmetric contributions add up little to the SM contribution, except for values between $\tan \beta \sim(10,20)$, where there could be both enhancing or suppressing effects. In Sec. III, we present the numerical anatomy of the $\operatorname{BR}\left(B_{s} \rightarrow\right.$ $\left.\mu^{+} \mu^{-}\right)$based on the MSSM-30 frame for which there are sources for $C P$ violation beyond the CKM. We shall 

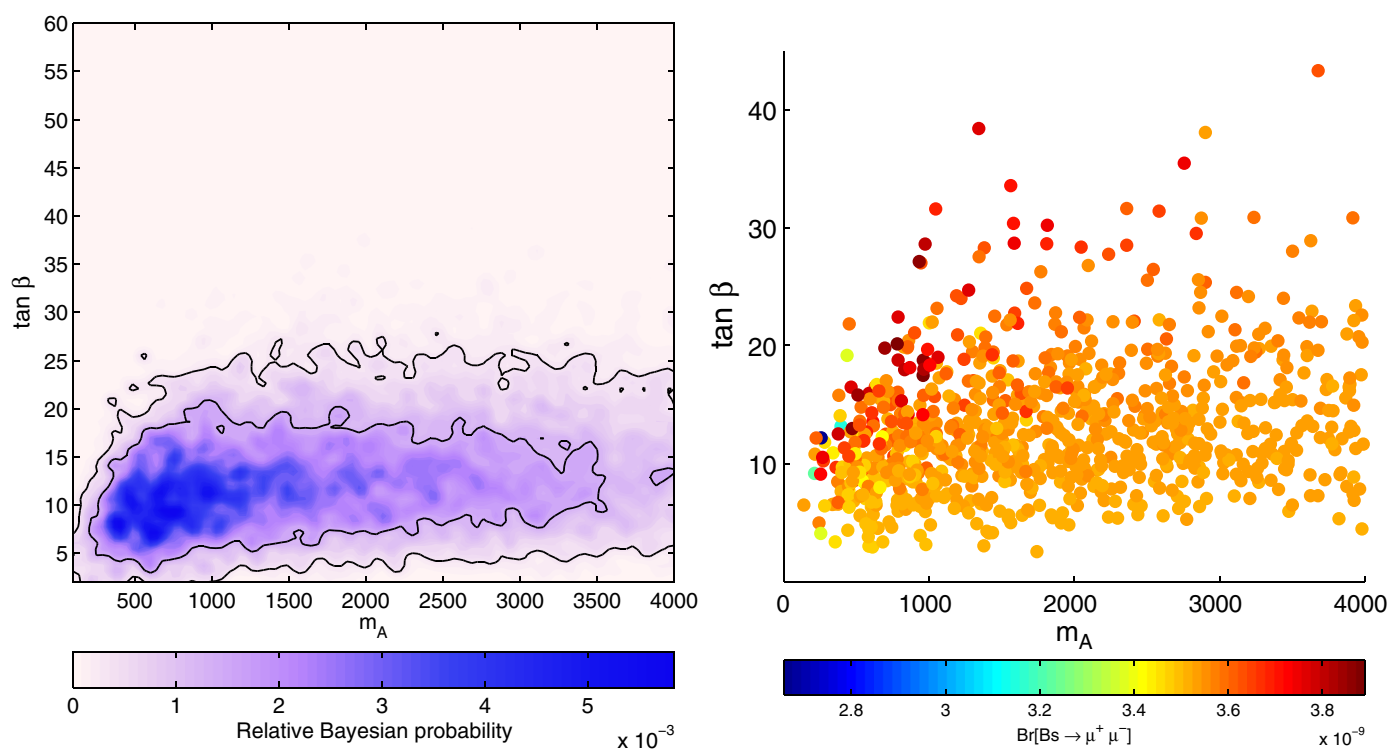

FIG. 2. Left: The marginalized 2D MSSM-30 $\left(m_{A}, \tan \beta\right)$ posterior distribution. The outer and inner contours enclose the $95 \%$ and $68 \%$ Bayesian probability regions respectively, $m_{A}$ is in a GeV scale. Right: The scatter plot on the same plane shows the correlation to $\operatorname{BR}\left(B_{s} \rightarrow \mu^{+} \mu^{-}\right)$without taking parameter points associated Bayesain posterior probabilities.

comment on the interplay of the contributions coming from the neutral Higgs, $H^{0}$ and $Z$ penguin diagrams after introducing the theory of supersymmetric contributions to $C_{S}, C_{P}$, and $C_{10}$. The box diagram contribution to any of the Wilson coefficients entering into $\operatorname{BR}\left(B_{s} \rightarrow \mu^{+} \mu^{-}\right)$is small in comparison to the SM [63]. Although this is strictly true in the case where the CKM matrix is the only source of $C P$ and flavor violation, in our case the contributions from the extra sources of $C P$ violation are generally negligible. We comment very briefly about the box contribution in Sec. III A.

For this work, the relevant posterior probability distribution, of the form of Eq. (7), which is a marginalised over the $2 \mathrm{D}\left(m_{A}, \tan \beta\right)$ plane is shown in Fig. 2. A 3D scatter plot showing the variations of $\operatorname{BR}\left(B_{s} \rightarrow \mu^{+} \mu^{-}\right)$on the same place is also shown. One of the aims of this article is to analyse the different contributions to the $\operatorname{BR}\left(B_{s} \rightarrow \mu^{+} \mu^{-}\right)$within the MSSM-30 posterior and to assess the impact of the recent $\operatorname{BR}\left(B_{s} \rightarrow \mu^{+} \mu^{-}\right)$measurement on the MSSM-30 parameters posterior. We shall address the latter case in what follows and the former in Sec. III. The posterior distribution in Fig. 2 (left) shows that high $\tan \beta$ values are disfavored. The result of the MSSM-30 global fit shows that $\tan \beta$ lies in the range 4.5 to 26.9 at $95 \%$ Bayesian probability interval. This feature is new compared to the pMSSM fits in [8,32]. The reason why the two distributions have different shape is mainly due to the inclusion of new $C P$-violating parameters in the MSSM-30 and the leptonic electric dipole moment constraints which tend to be proportional to $\tan \beta$ [72]. In order to limit the over production of the dipole moments, relatively lower, in comparison to the $\mathrm{pMSSM}, \tan \beta$ values are needed. In Fig. 2 (right), the weight-free scatter plot shows the correlations of $\operatorname{BR}\left(B_{s} \rightarrow \mu^{+} \mu^{-}\right)$along the $m_{A}$ or $\tan \beta$ directions within the global fit posterior. It can be seen that independently of $m_{A}$ above some few 100s of $\mathrm{GeV}$ and for $\tan \beta \sim 5$, the value of $\operatorname{BR}\left(B_{s} \rightarrow \mu^{+} \mu^{-}\right) \sim$ $3.5 \times 10^{-9}$ is constant. This indicates a possible tension between the MSSM-30 global fit posterior described here with the $\operatorname{BR}\left(B_{s} \rightarrow \mu^{+} \mu^{-}\right)$measurement [18] given that, for instance, assuming a future $\operatorname{BR}\left(B_{s} \rightarrow \mu^{+} \mu^{-}\right)$precision of $15 \%$ relative to the central value kills most of the posterior points and the surviving ones have sub-TeV $m_{A}$. This result is only indicative. A robust inference concerning the impact of such a plausible future $\operatorname{BR}\left(B_{s} \rightarrow\right.$ $\mu^{+} \mu^{-}$) precision will require new fits of the MSSM-30 to data. This is because the result and any other feature within the posterior sample is obviously due to the resultant effect of the various observables in Table I used for constraining the MSSM-30 parameters. The main message here is that current $\operatorname{BR}\left(B_{s} \rightarrow \mu^{+} \mu^{-}\right)$measurement [18] and possible future precisions will most likely reduce the allowed MSSM-30 region.

\section{MSSM-30 CONTRIBUTIONS TO $B_{s} \rightarrow \mu^{+} \mu^{-}$}

For the MSSM-30 there are new sources of flavor and $C P$ violation beyond the CKM and therefore the contribution from different particles becomes relevant. As it will be shown later, the neutralino and gluino contributions can compete with those from charginos. Although all of these contributions are suppressed in the MSSM-30 posterior sample with $\tan \beta$ typically less than 30 . Therefore, for making contrast to the various BSM contributions within the pMSSM and MSSM-30, different regimes for $\tan \beta$ are considered. 


\section{A. Diagram-by-diagram and particle-by-particle contributions}

From here on, we refer to the Box, Higgs penguin and $\mathrm{Z}$ penguin diagrams as kind of diagrams for which some examples are shown in Fig. 3. As mentioned in Sec. II C, $C_{10}$ in the SM gets its larger contribution from the $Z$ penguin with a top loop, about $75 \%$ and its second largest contribution from the $W$ box, 24\%. Higgs penguin contributions in the SM are highly suppressed. On the other hand, the largest contribution in the pMSSM comes from the second diagram of Fig. 3 since the degeneracy of scalar masses $\tilde{Q}$ is broken by radiative effects induced by Yukawa couplings. This produces and effective flavor off-diagonal piece which does not go away when rotating to the mass eigenstates basis [6]. In the MSSM-30 off-diagonal elements are present and compete with the contribution coming from the afore mentioned radiative effects. In the case of MSSM-30, contributions from the $Z$ penguin diagrams are in general suppressed, except for low value of $\tan \beta(\lesssim 10)$.

In Figs. 4 and 5 we compare the pMSSM and the MSSM-30 in terms of their contributions to $\operatorname{BR}\left(B_{s} \rightarrow\right.$ $\mu^{+} \mu^{-}$) coming from different diagrams and particles respectively. Here by "particles," we refer to "gluino," "chargino," "neutralino," and "W + charged Higgs boson," understanding that these particles can only come in their respective loops together with squarks type down, squarks
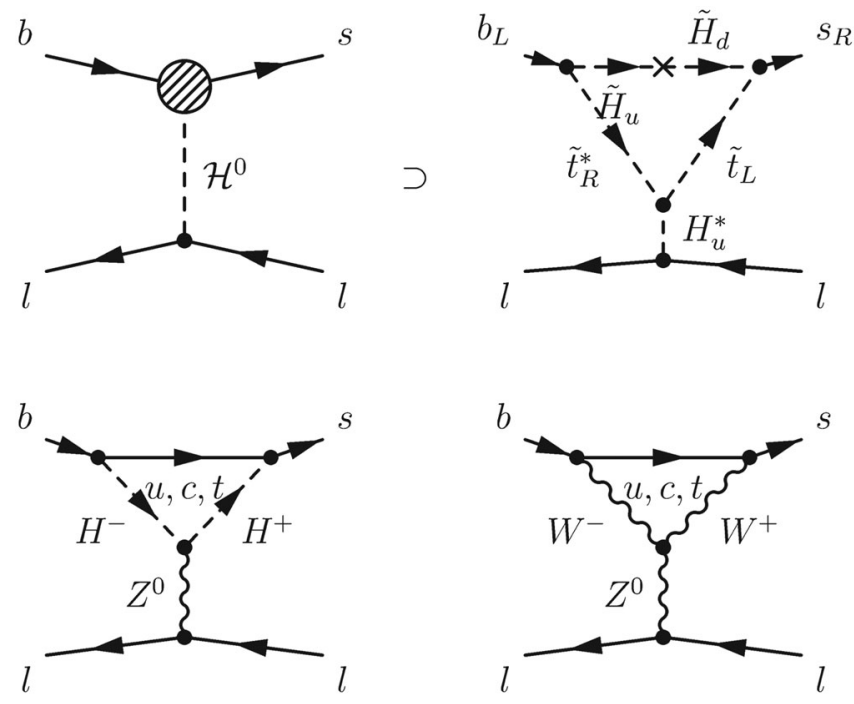

FIG. 3. Higgs penguin and $\mathrm{Z}$ penguin diagrams contributing to $\operatorname{BR}\left(B_{s} \rightarrow \mu^{+} \mu^{-}\right)$. The first diagram represents the general Higgs penguin diagram. The most important contribution in the pMSSM comes from the second diagram of the first line, depicted in flavor basis. Even in the pMSSM this is the most important contribution since the degeneracy of scalar masses $\tilde{Q}$ is broken by radiative effects induced by Yukawa couplings and hence induces and effective flavor off-diagonal piece which does not go away when rotating to mass eigenstates basis [6]. The last $\mathrm{Z}$ penguin gives the leading SM contribution. type up, squarks type down and quarks type up, respectively. The contributions for the pMSSM (solid black lines) are compared to the MSSM-30 case (red dashed lines). We can see that the distributions for the $\mathrm{Z}$ penguin and box diagrams become narrower in the MSSM-30, in comparison to those of the pMSSM, but overall these contributions shift $\operatorname{BR}\left(B_{s} \rightarrow \mu^{+} \mu^{-}\right)$to higher values than in the pMSSM case. For the MSSM-30, the contributions to the Higgspenguin diagrams become a bit suppressed, because of the preferred bigger masses for $m_{A}$ and $m_{\tilde{t}}$ and lower values for $\tan \beta$.

\section{B. Contributions to $C_{P}$ and $C_{S}$}

\section{1. $H^{0}$-penguin}

As mentioned above, the most important contribution in the pMSSM comes from the Higgs penguin diagram depicted in the second diagram of Fig. 3. This happens because the degeneracy of scalar masses $\tilde{Q}$ is broken by radiative effects induced by Yukawa couplings and hence this induces and effective flavor off-diagonal piece which does not go away when rotating to mass eigenstate basis [6]. The large $\tan \beta$ region of this contribution can play a very significant role. This can be understood by writing the simplified contribution at $\mathrm{LO}$ as [1]

$$
\begin{aligned}
C_{S}^{H^{0}}\left(\tilde{\chi}^{ \pm}\right) \approx & -C_{P}^{H^{0}}\left(\tilde{\chi}^{ \pm}\right) \\
= & \mu A_{t} \frac{\tan ^{3} \beta}{\left(1+\epsilon_{b} \tan \beta\right)^{2}} \frac{m_{t}^{2}}{m_{\tilde{t}}^{2}} \frac{m_{b} m_{\mu}}{4 M_{W}^{2} m_{A}^{2} \sin ^{2} \theta_{W}} \\
& \times x\left[\frac{(1-x)+\log (x)}{(1-x)^{2}}\right], \\
x= & m_{\tilde{t}}^{2} / m_{\tilde{\chi}_{1}^{ \pm}}^{2}, \quad m_{\tilde{\chi}_{1}^{ \pm}} \approx \mu,
\end{aligned}
$$

which is quite sensitive to $m_{A}$ and $m_{\tilde{t}}$ and therefore drops noticeably with the increase of their values. From this expression, we can also understand that the lower the value of $\tan \beta$, the lower the contribution to $\operatorname{BR}\left(B_{s} \rightarrow \mu^{+} \mu^{-}\right)$ from this diagram. Since for our fits, the preferred values of $m_{\tilde{t}}$ are $O(1) \mathrm{TeV}$ for the pMSSM, the suppression of this contribution becomes considerable.

In the MSSM-30, the off-diagonal parameters in the softsquared masses and trilinear terms, Eq. (1), add up to the contributions given by the broken degeneracy of the of scalar masses $\tilde{Q}$. In this case, the contributions to $C_{S, P}^{H^{0}}\left(\tilde{\chi}^{ \pm}\right)$ cannot be written in the form of Eq. (18), because nonzero off-diagonal terms are present even before the breaking of the degeneracy of the diagonal soft-squared masses. However, we find that the differences between the contributions of $C_{S, P}^{H^{0}}\left(\tilde{\chi}^{ \pm}\right)$in the pMSSM and in the MSSM-30 is only at the percent level. In this respect, in the MSSM-30, there could be cancellations among these two contributions for equally heavy/high magnitude parameters (e.g., $\mu$ and $\left.A_{t}\right)$. These cancellations could make the $\operatorname{BR}\left(B_{s} \rightarrow \mu^{+} \mu^{-}\right)$ 

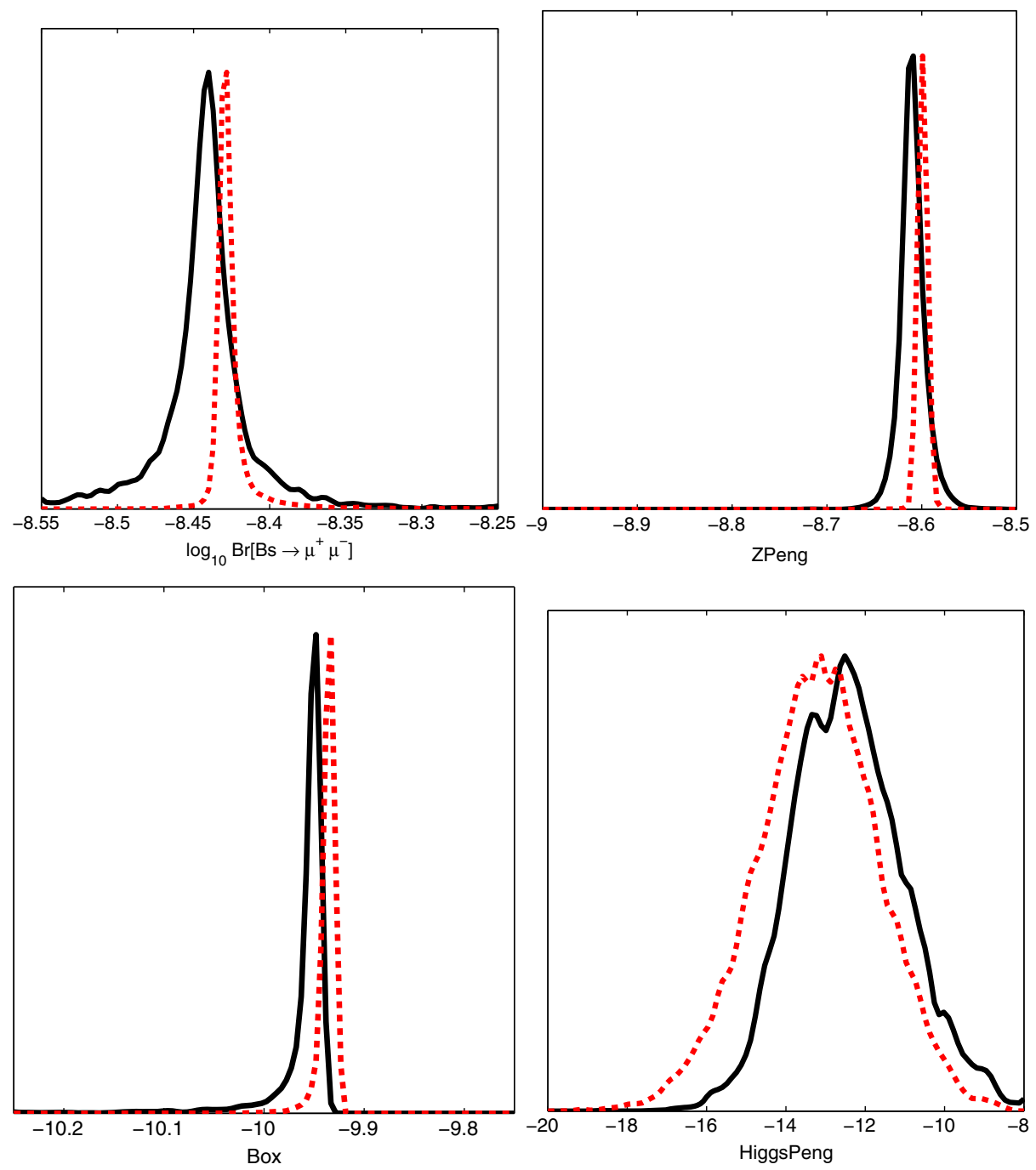

FIG. 4. Contribution by diagrams to $\operatorname{BR}\left(B_{s} \rightarrow \mu^{+} \mu^{-}\right)$. The solid black lines correspond to the pMSSM and the dashed red lines to the MSSM-30. The distributions for the Z penguin and box diagrams become narrower in the MSSM-30 in comparison to those of the pMSSM, but overall these contributions shift $\operatorname{BR}\left(B_{s} \rightarrow \mu^{+} \mu^{-}\right)$to higher values than in the pMSSM case. For the MSSM-30, the contributions to the Higgs-penguin diagrams become suppressed, due to the preferred bigger masses for $m_{A}$ and $m_{\tilde{t}}$ and lower values for $\tan \beta$. For all plots, the vertical axis represent the relative probability density associated to a point in the MSSM scan. The horizontal axis is the logarithm of the corresponding contribution to $\operatorname{BR}\left(B_{s} \rightarrow \mu^{+} \mu^{-}\right)$.

independent of $m_{A}$ as shown in Fig. 1 (top-right), and make the contribution to the Wilson Coefficients not so different from the pMSSM.

\section{Z-penguin and Box diagrams}

Supersymmetric particles propagating in the loop cannot generate a contributions to $C_{S, P}, C_{S, P}^{\prime}$ due to the vector coupling to $Z^{0}$. Diagrams with charginos propagating in the box also give rise to non zero values of $C_{S}^{\chi}$ and $C_{P}^{\chi}$ but leave $C_{S}^{\prime \chi}=C_{P}^{\prime \chi}=0$. In the case where the masses of squark and sneutrino in the box are degenerate, one can have $C_{S}^{\chi}=-C_{P}^{\chi}$. Diagrams with one charged Higgs boson propagating in the box can give non-zero contributions only through the left-handed parts so that $C_{S}^{H^{+}}=-C_{P}^{H^{+}}$and $C_{S}^{\prime H^{+}}=-C_{P}^{\prime H^{+}}$[73].

\section{Contributions to $C_{10}$}

\section{Z-penguin}

For heavy charged Higgs bosons and low values of $\tan \beta \leq 20$, Higgs penguin and box diagrams are small. Hence, the contribution from the $Z$ (third diagram in Fig. 3) penguin becomes the dominant one among all of the contributions to $C_{10}$. This effect becomes accentuated for very small values of $\tan \beta$.

Our fits favor intermediate values of $\tan \beta$, so in principle there is a transition between the regimes of $H^{0}$ and $Z$ penguin dominance. However, this also depends on the values of the charged-Higgs mass and most importantly on the value of $m_{H}$, being large, there is not a surprise that both contributions are mostly 

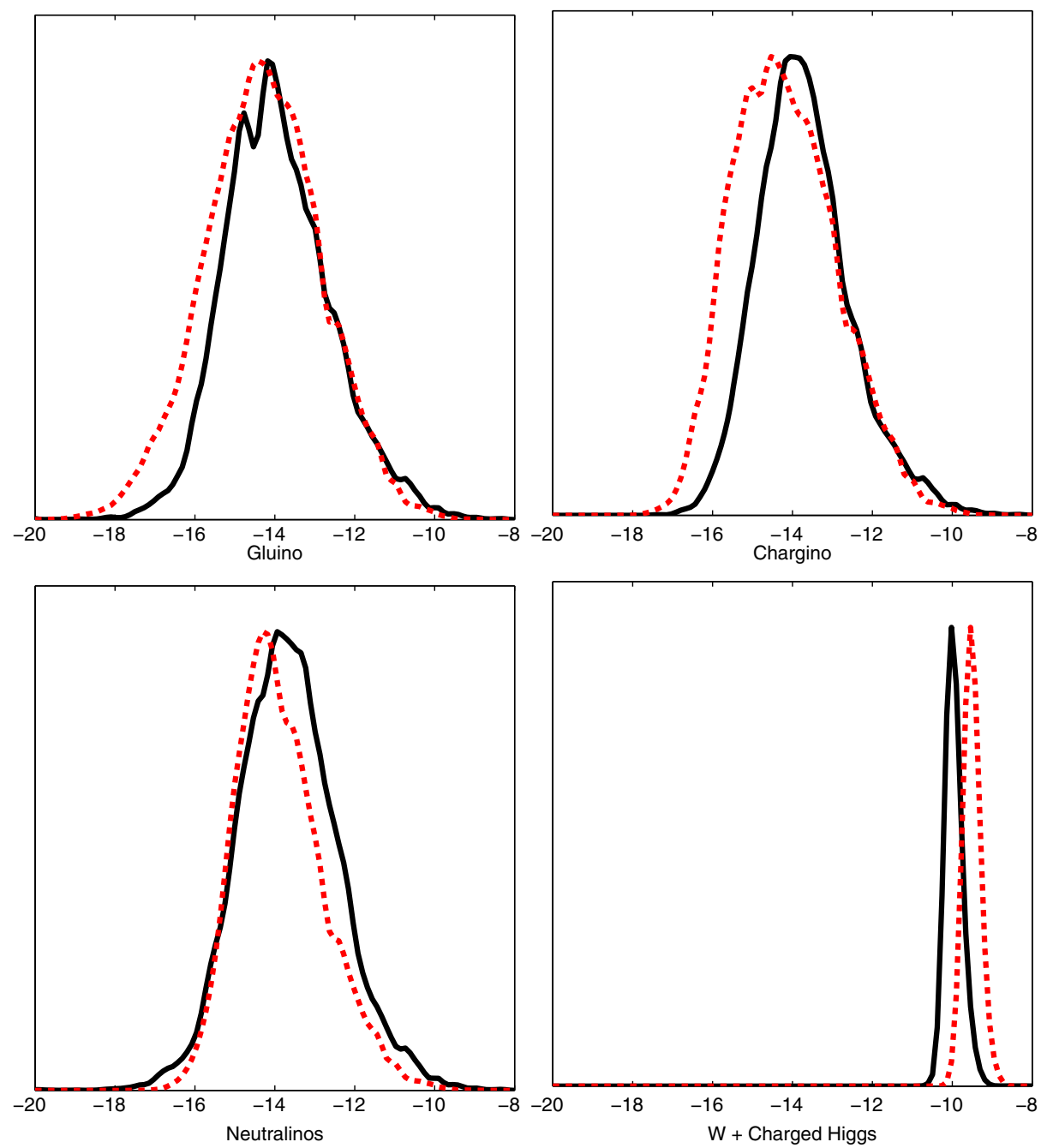

FIG. 5. The particle-by-particle contributions to $\operatorname{BR}\left(B_{s} \rightarrow \mu^{+} \mu^{-}\right)$. The solid black lines correspond to the pMSSM and the dashed red lines to the MSSM-30. In SUSY_FLAVOR the charged-Higgs contribution cannot be separated from the SM W-boson contribution. The axes are as in Fig. 3.

suppressed. At LO, the corresponding contributions to $C_{10}$ and $C_{10}^{\prime}$ are [1]

$$
\begin{aligned}
C_{10}^{Z}\left(H^{ \pm}\right) & =\frac{1}{8 \sin _{W}^{2}} \frac{m_{t}^{2}}{m_{W}^{2}} \frac{1}{\tan ^{2} \beta} f_{H}\left(y_{t}\right), \\
C_{10}^{\prime Z}\left(H^{ \pm}\right) & =-\frac{1}{8 \sin _{W}^{2}} \frac{m_{s} m_{b}}{m_{W}^{2}} \tan ^{2} \beta f_{H}\left(y_{t}\right), \\
f_{H}\left(y_{t}\right) & =\frac{y_{t}}{1-y_{t}}\left(1+\frac{1}{1-y_{t}} \log y_{t}\right), \\
y_{t} & =m_{t}^{2} / m_{H^{-}}^{2}
\end{aligned}
$$

Using SUSY_FLAVOR we find that the total contribution to $C_{10}$ is $C_{10 T}^{Z}\left(H^{ \pm}\right)=C_{10}^{Z}\left(H^{ \pm}\right)-C_{10}^{\prime Z}\left(H^{ \pm}\right) \in\left(O\left(10^{-3}\right)\right.$, $\left.O\left(10^{-2}\right)\right)$, adding up little to the total of $C_{10}$, including the SM contributions. For our set of experimental values, we obtain that $C_{10}^{\mathrm{SM}}=-4.13 \pm 0.05$. In the $\mathrm{SM}$ the current accuracy for this coefficient is better than $0.1 \%$ level when allowing only the top-quark mass and the strong coupling constant to deviate from their corresponding central value [69]. In principle, then the contributions from supersymmetric particles could be disentangled from the SM uncertainty.

For illustration of our discussion, in Fig. 6 we have made a comparison at $\mathrm{LO}$ of $C_{10 T}^{Z}\left(H^{ \pm}\right)$and $C_{S}^{H^{0}}\left(\chi^{ \pm}\right)$to emphasise the importance of the values of $m_{H^{-}}$and $\tan \beta$ in order to determine from which kind of diagram the contributions to $\operatorname{BR}\left(B_{s} \rightarrow \mu^{+} \mu^{-}\right)$are the most important for each of the samples, the pMSSM and the MSSM-30. This comparison is made in the right panel of the figure, while in the left panel we show only cases which correspond to the MSSM-30 sample. Although in general for heavy spectra, both contributions are really small in comparison to the SM contributions, one can still appreciate the relevance of some supersymmetric particles. The solid lines correspond to $C_{10 T}^{Z}\left(H^{ \pm}\right)$and the dashed and dot-dashed to $C_{S}^{H^{0}}\left(\chi^{ \pm}\right)$. 

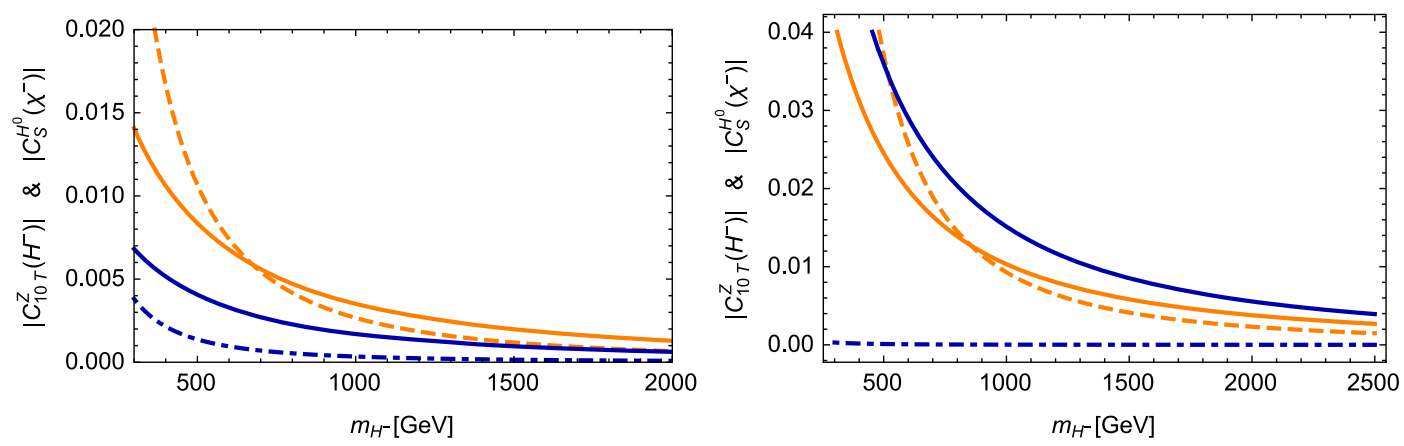

FIG. 6. $\left|C_{10 T}^{Z}\left(H^{ \pm}\right)\right|$and $\left|C_{S}^{H^{0}}\left(\chi^{ \pm}\right)\right|$as a function of $m_{H^{ \pm}}\left(\approx m_{A}\right)$ for typical values of the pMSSM and the MSSM-30 samples. The solid lines correspond to $\left|C_{10 T}^{Z}\left(H^{ \pm}\right)\right|$and the dashed and dot-dashed to $\left|C_{S}^{H^{0}}\left(\chi^{ \pm}\right)\right|$. For both plots, $\left(A_{t}, m_{\tilde{t}}, \mu\right)=(1200,1200,100) \mathrm{GeV}$. For the left panel, the two values of $\tan \beta$ are 25 (high) and 12 (low), are plotted in orange-light- and dark-blue- respectively. These values correspond respectively to the typical values for the pMSSM and the MSSM-30 sample. For the right panel, we plotted the extreme values of the MSSM-30 sample, $\tan \beta=40,5$, plotted in orange-light- and dark-blue, respectively. In this last plot, we can appreciate the enhancement of $\left|C_{10 T}^{Z}\left(H^{ \pm}\right)\right|$for small values of $\tan \beta$.

For both plots, $\left(A_{t}, m_{\tilde{t}}, \mu\right)=(1200,1200,100) \mathrm{GeV}$. For the left panel the two values of $\tan \beta$ are 25 (high) and 12 (lower). These values correspond respectively to the typical values for the pMSSM and the MSSM-30 sample. For the right panel, we plotted the extreme values of the MSSM-30 sample, $\tan \beta=5,40$.

We can see that, as it is well established, for values of $m_{H^{-}}$below $1 \mathrm{TeV}$, the contribution from the large $\tan \beta$ values (here 25) coming from $C_{S}^{H^{0}}\left(\chi^{ \pm}\right)$is the leading one (in Fig. 6 represented by the orange-light- dashed line). On the other hand, contributions to both $C_{10 T}^{Z}\left(H^{ \pm}\right)$and $C_{S}^{H^{0}}\left(\chi^{ \pm}\right)$for values of $\tan \beta$ around 10 are typically less than a third of the corresponding values when $\tan \beta>20$. Since for the pMSSM sample, values for $\tan \beta$ above 20 dominate the sample, it is clear that the most important contributions come from $C_{S}^{H^{0}}\left(\chi^{ \pm}\right)$. For the MSSM-30 sample however, smaller values than 10 for $\tan \beta$ are an important part of the sample and in this case they can give the largest supersymmetric contribution to the Wilson coefficients via $C_{10 T}^{Z}\left(H^{ \pm}\right)$. This is appreciated in the plot of the left in Fig. 6 where $C_{10 T}^{Z}\left(H^{ \pm}\right)$for $\tan \beta=5$ and $m_{H^{-}}>700 \mathrm{GeV}$ is the dominant of the supersymmetric contributions (solid blue-dark line).

\section{Interplay of Wilson coefficients}

In order to understand the different contributions to Eq. (8), it is customary to compare the relative size of the Wilson Coefficients $C_{S}$ and $C_{P}$ to $C_{10}$. This is useful because $C_{S}$ and $C_{P}$ can only have supersymmetric contributions and supersymmetric contributions to $C_{10}$ are highly suppressed.

In order to do this comparison, we compare the value of $C_{10}$ to the Wilson Coefficients $C_{S}$ and $C_{P}$ but weighted in the same way that $C_{10}$ contributes to $\operatorname{BR}\left(B_{s} \rightarrow \mu^{+} \mu^{-}\right)$, Eq. (8). This allows a direct comparison among $C_{S}, C_{P}$, and $C_{10}$. The weighted Wilson coefficients $C_{S}$ and $C_{P}$ are respectively denoted by $\hat{C}_{S}$ and $\hat{C}_{P}$ :

$$
\begin{aligned}
\hat{C}_{S} & \equiv \frac{m_{B_{s}}^{2}}{2 m_{\mu} m_{b}} \sqrt{1-\frac{4 m_{\mu}^{2}}{m_{B_{s}}^{2}}} C_{S} \\
\hat{C}_{P} & \equiv \frac{m_{B_{s}}^{2}}{2 m_{\mu} m_{b}} C_{P} .
\end{aligned}
$$

In Fig. 7, we plot our results in planes $C_{10}$ vs $\hat{C}_{S}$ (we do not present $C_{10}$ vs $\hat{C}_{P}$ since $\hat{C}_{s} \approx-\hat{C}_{P}$ ), comparing the Wilson coefficients for the two samples. For the pMSSM sample we present only the results when fixing the top mass value, since for our MSSM-30 sample the top mass was kept fixed. In this sense, even if the sample is not complete for the pMSSM, we give a fair comparison to the MSSM-30 sample and we can be sure that the increase (in absolute value) of the Wilson coefficient $C_{10}$ arises due to the supersymmetric contributions. While in the pMSSM, the center value of $C_{10}$ is -4.61 in the MSSM-30 is -4.64 . Overall the contour plots for $C_{10}$ vs $C_{S}$ and $C_{10}$ vs $C_{P}$ cover a bigger area in the pMSSM (see also, e.g., [9]) than in the MSSM-30 but the one and two sigma regions of this last sample are shifted to the left, Fig. 7. As mentioned in Sec. III B, $C_{10}^{\mathrm{SM}}=-4.13 \pm 0.05$ and the current accuracy is of the order $0.1 \%$. Hence supersymmetric contributions could be also disentangled from the SM error.

From Fig. 6 we can see that for large values of $m_{H^{-}}$ $(>1500 \mathrm{GeV})$ the contributions from $\left|C_{10 T}^{Z}\left(H^{ \pm}\right)\right|$and $\mid C_{S}^{H^{0}}\left(\chi^{ \pm}\right)$become quite similar, especially for lower values of $\tan \beta$ (approximately below 20). On the other hand, since for the pMSSM sample, values for $\tan \beta$ above 20 dominate the sample, the most important contributions come from $C_{S}^{H^{0}}\left(\chi^{ \pm}\right)$. For the MSSM-30 sample however, smaller values than 10 for $\tan \beta$ are an important part of the sample and in this case they can give the largest supersymmetric contribution to the Wilson coefficients via $C_{10 T}^{Z}\left(H^{ \pm}\right)$, as mentioned before (Fig. 6).

We recall the reader that the present work builds further on the project for MSSM explorations within systematically 

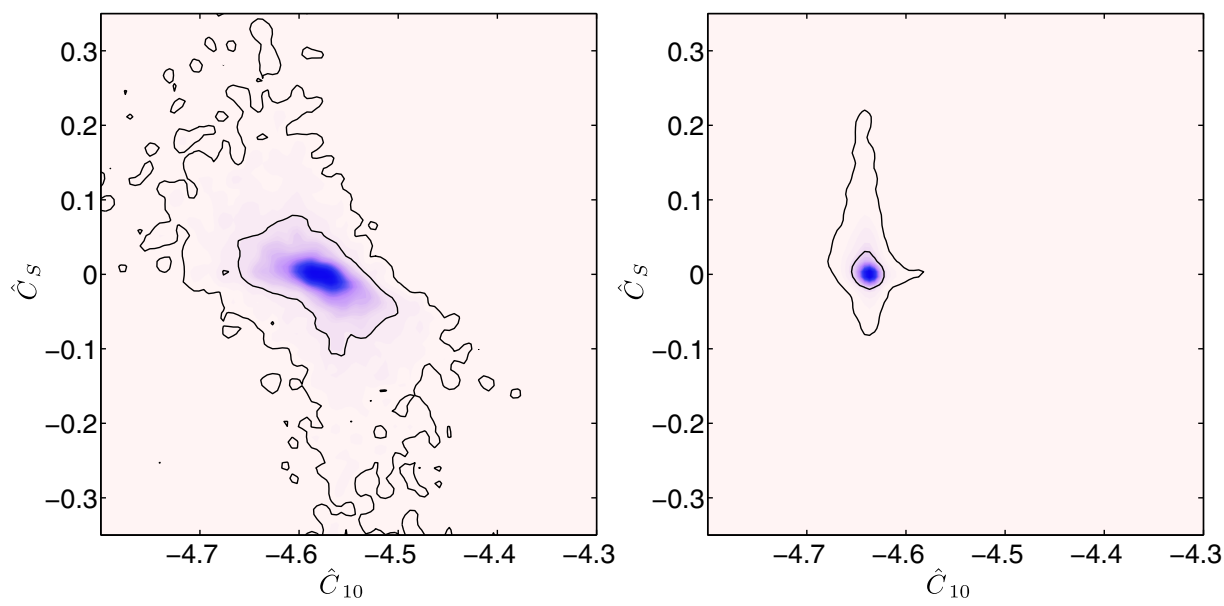

FIG. 7. We compare here the Wilson coefficients $C_{10}, C_{S}$, and $C_{P}$ for the pMSSM sample (left) and for the MSSM-30 sample (right). Within the pMSSM, $C_{10}=-4.58 \pm 0.06$ ranging from -4.68 to -4.50 at $95 \%$ Bayesian probability region. For the MSSM- 30 , $C_{10}=-4.64 \pm 0.01$ ranging from -4.66 to -4.63 at $95 \%$ Bayesian probability region.
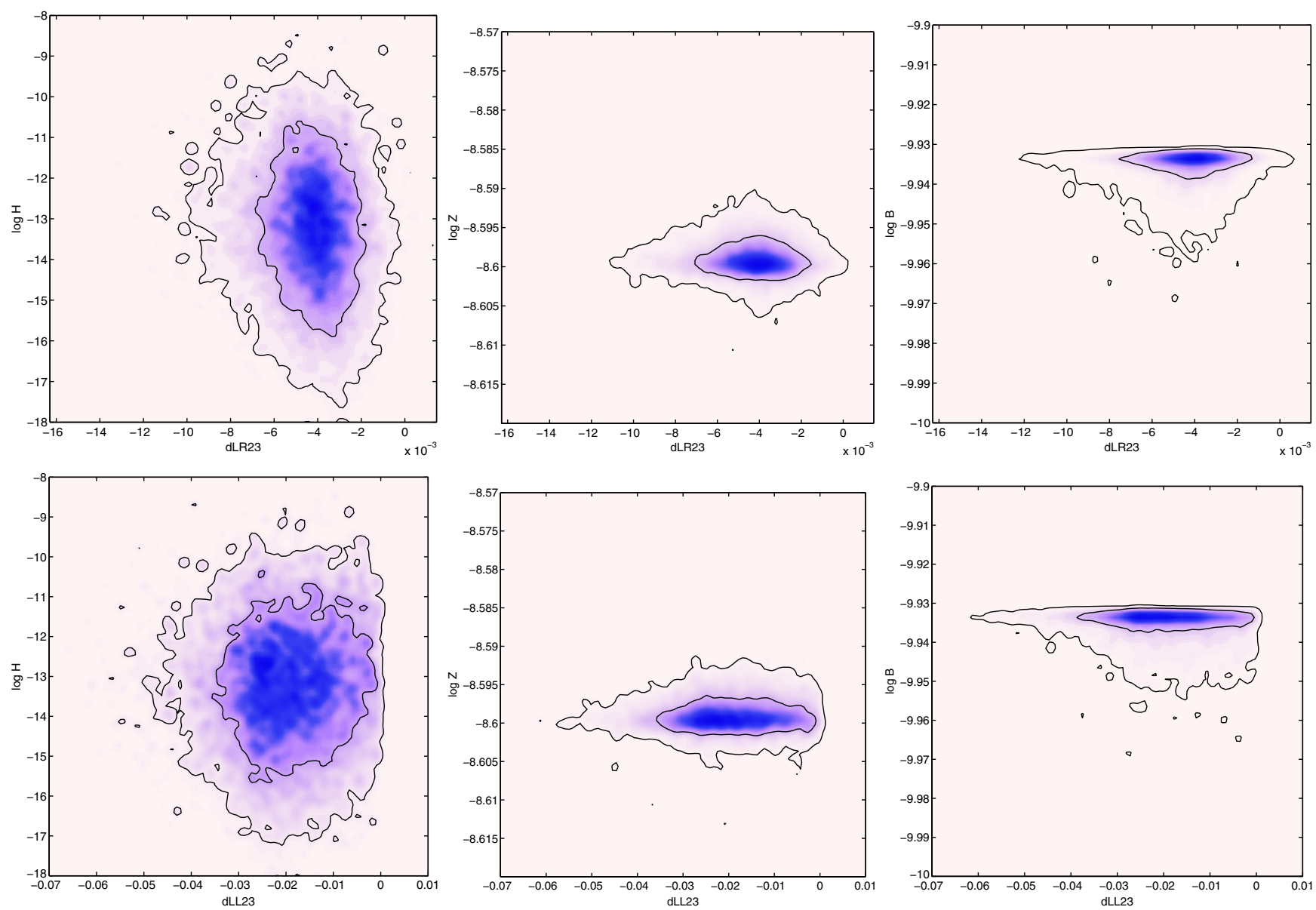

FIG. 8. Contributions to $\operatorname{BR}\left(B_{s} \rightarrow \mu^{+} \mu^{-}\right)$from the parameters $\mathrm{dLR} 23=\left(\delta_{O L R}\right)^{23}$ and dLL23 $=\left(\delta_{O L L}\right)^{23}$ (top and bottom rows respectively). $\log \mathrm{H}, \log \mathrm{Z}$, and $\log \mathrm{B}$ represent respectively the logarithms of the absolute values of Higgs Penguin, $\mathrm{Z}$ penguin and Box contributions to the total $\operatorname{BR}\left(B_{s} \rightarrow \mu^{+} \mu^{-}\right)$. The outer and inner contours enclose the $95 \%$ and $68 \%$ Bayesian probability regions, respectively. 
built frameworks, in this case a specific frame for flavor violation and a Bayesian approach for deriving inference from experimental data. The specific flavor violation determined by the MSSM-30 is a realistic one, owning to the fact that it can be understood as taking into account the running of off-diagonal elements of soft-squared masses and trilinears. In contrast, the pMSSM sets these elements to zero to start with. Since there are many supersymmetric contributions in a less constrained MSSM, it is natural to expect that these contributions will have a constructive interference instead of a destructive one, resulting in the increase of the value of $B_{s} \rightarrow \mu^{+} \mu^{-}$. This is effectively what is happening in the MSSM-30 in comparison to the pMSSM: owning to the fact that the MSSM-30 is less constrained, it does increase the value of $B_{s} \rightarrow \mu^{+} \mu^{-}$in comparison to the pMSSM. It is out of the scope of this work to identify a region where actually cancellations could take place, but it is definitely a project that it should be performed.

\section{E. Interplay of off-diagonal soft-squared elements}

It is customary to assess the impact of flavor violation in terms of the flavor violating parameters

$$
\delta_{Q X Y}^{i j}=\frac{\left(\hat{M}_{Q}^{2}\right)_{X Y}^{i j}}{\sqrt{\left(\hat{M}_{Q}^{2}\right)_{X X}^{i i}\left(\hat{M}_{Q}^{2}\right)_{Y Y}^{j j}}},
$$

which measure the amount of off-diagonal allowed contributions constrained by all relevant flavor observables (see Table 1 of [23]). Here $\left(\hat{M}_{Q}^{2}\right)_{L R}=-A_{D} v_{D}+\mu \tan \beta m_{D}$ $\left(v_{D}=v_{U} / \tan \beta\right.$ and $m_{D}$ the diagonal mass matrix of $\mathrm{D}$ quarks), $\left(\hat{M}_{Q}^{2}\right)_{R R}=M_{D}^{2},\left(\hat{M}_{Q}^{2}\right)_{L L}=M_{Q}^{2}$, the mass matrices without hat are those appearing in Eq. (2). Here we present only the relevant parameters for $\operatorname{BR}\left(B_{s} \rightarrow \mu^{+} \mu^{-}\right)$. In order to make manifest how the $\mathrm{Z}$ penguin contributions dominate the supersymmetric contributions of the coefficient $C_{10}$ for most part of the parameter space. We present in Fig. 8 (top row) the individual contributions to $\operatorname{BR}\left(B_{s} \rightarrow\right.$ $\mu^{+} \mu^{-}$) from Higgs penguin, $\mathrm{Z}$ penguin and box diagrams, as a function of $\left(\delta_{Q L R}\right)^{23}$. In this figure, we can clearly appreciate how $\mathrm{Z}$ penguin contributions are in general the most dominant for practically all values of $\left(\delta_{Q L R}\right)^{23}$ and as emphasized in [63], for small values of $\tan \beta(\leq 20)$, Higgs penguin contributions are small. Box contributions are also small, but they tend to be even bigger than the Higgs penguin contributions. In the second row of Fig. 8 we represent the individual contributions to $\operatorname{BR}\left(B_{s} \rightarrow \mu^{+} \mu^{-}\right)$ from Higgs penguin, $\mathrm{Z}$ penguin and Box diagrams, as a function of $\left(\delta_{Q L L}\right)^{23}$. As it is usual with LR flavor violation, it tends to be more constrained, as in this case it is allowed to be only $O\left(10^{-3}\right)$, while LL flavor violation it can be of $O\left(10^{-2}\right)$.

\section{CONCLUSIONS}

We have continued with our studies in order to explore features of the MSSM by using Bayesian statistical techniques on systematically constructed, symmetry-guided, MSSM frameworks beyond the traditional constructions. Here, the phenomenological framework considered is the 30-parameter-MSSM, called MSSM-30, and the observable of interest is the $B_{s} \rightarrow \mu^{+} \mu^{-}$decay. The measured branching ratio $\operatorname{BR}\left(B_{s} \rightarrow \mu^{+} \mu^{-}\right)$is compatible with the $\mathrm{SM}$ prediction but it still has a large (order 20\%) uncertainty. Future precision measurements of this observable will be excellent for assessing the MSSM as new physics beyond the SM. Within the MSSM-30 a posterior sample was considered, for which a $15 \%$ uncertainty on the measured $\operatorname{BR}\left(B_{s} \rightarrow \mu^{+} \mu^{-}\right)$ would favour a sub-TeV pseudoscalar Higgs boson.

Knowing that the decay $B_{s} \rightarrow \mu^{+} \mu^{-}$is a good indicator for assessing models with extended Higgs sectors, we compared the MSSM-30 to the pMSSM to see if there are any physics or features in the parameters of the former which are not accessible in the latter. It turned out to be the case since the MSSM-30 sample prefers lower values of $\tan \beta \sim(10,20)$ in comparison to the pMSSM which prefers $\tan \beta \sim(20,40)$. It is then possible to find higher values for $\operatorname{BR}\left(B_{s} \rightarrow \mu^{+} \mu^{-}\right)$in the MSSM-30 mainly due to bigger contributions coming from diagrams involving charginos and Z-penguin diagrams.

We found that the best way to analyse the contributions to the branching ratio $\operatorname{BR}\left(B_{s} \rightarrow \mu^{+} \mu^{-}\right)$was by comparing kind of diagrams: $Z$ penguin, box, and Higgs penguin diagrams, instead of comparing contributions of supersymmetric particles (i.e., gluinos, neutralinos or charginos). The reason is that in the pMSSM the supersymmetric contributions become quite suppressed due to the large values of $m_{A}$ and $m_{\tilde{t}}$ (well into the multi-TeV region) and that in the SM the $Z$ penguin and box contributions are dominant ( $\sim 75 \%$ and $24 \%$ respectively). This last fact then in principle helps to look for contributions coming from supersymmetry. In the pMSSM it is well established that the major $\mathrm{BSM}$ contributions to $\operatorname{BR}\left(B_{s} \rightarrow \mu^{+} \mu^{-}\right)$come from a Higgs penguin and a chargino in the loop. Hence, this is a specific example of how analysis by kind of diagrams becomes relevant.

The MSSM-30 has by construction nonzero off-diagonal soft-squared mass terms, contrary to the pMSSM where they are set to zero by hand. When analyzing both samples using a Bayesian fit, both samples are constrained by the same observables. Therefore, if no cancellations appear, the value of the allowed effective off-diagonal soft-squared mass terms should be of the same order in both samples. The best place to look for the difference between both samples is in the contribution coming from the charginos, mainly from Higgs-penguins, (Fig. 5) where we can see that the distributions from PMSSM and MSSM-30 are different but mostly indistinguishable towards higher values of $\operatorname{BR}\left(B_{s} \rightarrow \mu^{+} \mu^{-}\right)$. 
In order to assess the impact of BSM contributions to B observables, it is customary to compare the size of the Wilson coefficients which receive the majority of the BSM contributions with the Wilson coefficients present only in the SM case. For the decay $B_{s} \rightarrow \mu^{+} \mu^{-}$, the relevant coefficients are $C_{S}$ and $C_{P}$, which are scalar operators sensitive to the chirality of BSM contributions. In the SM the only contribution comes from the vector operator $C_{10}^{\mathrm{SM}}$, for which we find a value of $-4.13 \pm 0.05$. We have compared the coefficients $C_{S}$ and $C_{P}$ to $C_{10}$, using $C_{10}$ vs $C_{S}$ and $C_{10}$ vs $C_{P}$ planes and found that in the MSSM-30, $C_{S}$ and $C_{P}$ represent typically only a $\mathrm{O}(1 \%)$ contribution to the branching ratio $B_{s} \rightarrow \mu^{+} \mu^{-}$. Within the pMSSM $C_{10}=$ $-4.58 \pm 0.06$ ranging from -4.68 to -4.50 at $95 \%$ Bayesian probability region. For the MSSM-30, $C_{10}=$ $-4.64 \pm 0.01$ ranging from -4.66 to -4.63 at $95 \%$ Bayesian probability region. The MSSM-30 is more severely away from the SM value compared to the pMSSM. The current SM accuracy in determining the value of $C_{10}$ is of the order $0.1 \%$ and therefore supersymmetric contributions can be disentangled from the SM uncertainty.
Finally, as an outlook we highlight that future improvements of the measurement of $\operatorname{BR}\left(B_{s} \rightarrow \mu^{+} \mu^{-}\right)$, along with the measurement of other observables, like $\mathcal{A}_{\Delta \Gamma}^{\mu \mu}$ and $\operatorname{BR}\left(B_{d} \rightarrow \mu^{+} \mu^{-}\right)$which are correlated with $\operatorname{BR}\left(B_{s} \rightarrow \mu^{+} \mu^{-}\right)$, will play a crucial role in shaping the parameter space of the MSSM-30. On the other hand, systematically constructed frameworks which can capture the flavor structure of the MSSM, like the one presented here, should be favored over simplified scenarios which cannot capture the rich flavor structure of the MSSM.

\section{ACKNOWLEDGMENTS}

We would like to thank Janusz Rosiek for help and comments regarding SUSY_FLAVOR. L. V. S. thanks R. Fleischer for comments regarding $\operatorname{BR}\left(B_{s} \rightarrow \mu^{+} \mu^{-}\right)$and the Abdus Salam International Center for Theoretical Physics, Italy, for support and hospitality during the last stages of this project. During the initial stage of research leading to these results, S. S. A. received funding from the European Research Council under the European Union's Seventh Framework Programme (FP/2007-2013)/Grant No. 279972.
[1] C. Bobeth, T. Ewerth, F. Kruger, and J. Urban, Analysis of neutral Higgs boson contributions to the decays $\bar{B}(s) \rightarrow$ $\ell^{+} \ell^{-}$and $\bar{B} \rightarrow K \ell^{+} \ell^{-}$, Phys. Rev. D 64, 074014 (2001).

[2] C. Bobeth, T. Ewerth, F. Kruger, and J. Urban, Enhancement of $B\left(B_{d} \rightarrow \mu^{+} \mu^{-}\right) / B\left(B_{s} \rightarrow \mu^{+} \mu^{-}\right)$in the MSSM with minimal flavor violation and large tan beta, Phys. Rev. D 66, 074021 (2002).

[3] C. S. Huang, W. Liao, and Q. S. Yan, Promising process to distinguish supersymmetric models with large $\tan \beta$ from the standard model: $\vec{B} X_{s \mu^{+} \mu^{-}}$, Phys. Rev. D 59, 011701 (1998).

[4] C. Hamzaoui, M. Pospelov, and M. Toharia, Higgs mediated FCNC in supersymmetric models with large tan Beta, Phys. Rev. D 59, 095005 (1999).

[5] S. R. Choudhury and N. Gaur, Dileptonic decay of $B_{s}$ meson in SUSY models with large $\tan \beta$, Phys. Lett. B 451, 86 (1999).

[6] K. S. Babu and C. F. Kolda, Higgs Mediated $B^{0} \rightarrow \mu^{+} \mu^{-}$in Minimal Supersymmetry, Phys. Rev. Lett. 84, 228 (2000).

[7] C.S. Huang, W. Liao, Q.S. Yan, and S. H. Zhu, $B_{s} \rightarrow$ lepton + lepton-in a general 2 HDM and MSSM, Phys. Rev. D 63, 114021 (2001); Erratum, Phys. Rev. D64, 059902(E) (2001).

[8] S. S. AbdusSalam, B. C. Allanach, F. Quevedo, F. Feroz, and M. Hobson, Fitting the phenomenological MSSM, Phys. Rev. D 81, 095012 (2010).

[9] A. Arbey, M. Battaglia, F. Mahmoudi, and D. Martnez Santos, Supersymmetry confronts $B_{s} \rightarrow \mu^{+} \mu^{-}$: Present and future statusPhys. Rev. D 87, 035026 (2013).
[10] W. Altmannshofer, C. Niehoff, and D. M. Straub, $B_{s} \rightarrow$ $\mu^{+} \mu^{-}$as current and future probe of new physics, J. High Energy Phys. 05 (2017) 076.

[11] T. Aaltonen et al. (CDF Collaboration), Search for $B_{s}^{0} \rightarrow$ $\mu^{+} \mu^{-}$and $B^{0} \rightarrow \mu^{+} \mu^{-}$Decays with CDF II, Phys. Rev. Lett. 107, 191801 (2011); Erratum, Phys. Rev. Lett.107, 239903 (2011).

[12] V. M. Abazov et al. (D0 Collaboration), Search for the rare decay $B_{s}^{0} \rightarrow \mu^{+} \mu^{-}$, Phys. Lett. B 693, 539 (2010).

[13] R. Aaij et al. (LHCb Collaboration), Strong Constraints on the Rare Decays $B_{s} \rightarrow \mu^{+} \mu^{-}$and $B^{0} \rightarrow \mu^{+} \mu^{-}$, Phys. Rev. Lett. 108, 231801 (2012).

[14] S. Chatrchyan et al. (CMS Collaboration), Search for $B_{s}^{0} \rightarrow$ $\mu^{+} \mu^{-}$and $B^{0} \rightarrow \mu^{+} \mu^{-}$decays, J. High Energy Phys. 04 (2012) 033.

[15] G. Aad et al. (ATLAS Collaboration), Search for the decay $B_{s}^{0} \rightarrow \mu \mu$ with the ATLAS detector, Phys. Lett. B 713, 387 (2012).

[16] R. Aaij et al. (LHCb Collaboration), First Evidence for the Decay $B_{s}^{0} \rightarrow \mu^{+} \mu^{-}$, Phys. Rev. Lett. 110, 021801 (2013).

[17] V. Khachatryan et al. (CMS and LHCb Collaborations), Observation of the rare $B_{s}^{0} \rightarrow \mu^{+} \mu^{-}$decay from the combined analysis of CMS and LHCb data, Nature (London) 522, 68 (2015).

[18] M. Mulder (LHCb Collaboration), The branching fraction and effective lifetime of $B_{(s)}^{0} \rightarrow \mu^{+} \mu^{-}$at LHCb with Run 1 and Run 2 data, arXiv:1705.03274.

[19] K. Kadota, G. Kane, J. Kersten, and L. Velasco-Sevilla, Flavor issues for string-motivated heavy scalar spectra with 
a low gluino mass: The G2-MSSM case, Eur. Phys. J. C 72, 2004 (2012).

[20] J. Ellis, K. Olive, and L. Velasco-Sevilla, Maximal sfermion flavor violation in super-GUTs, Eur. Phys. J. C 76, 562 (2016).

[21] Z. Poh and S. Raby, Yukawa unification in an SO(10) SUSY GUT: SUSY on the edge, Phys. Rev. D 92, 015017 (2015).

[22] G. Colangelo, E. Nikolidakis, and C. Smith, Supersymmetric models with minimal flavor violation and their running, Eur. Phys. J. C 59, 75 (2009).

[23] S. S. AbdusSalam, C. P. Burgess, and F. Quevedo, MFV reductions of MSSM parameter space, J. High Energy Phys. 02 (2015) 073.

[24] A. Dedes, H. K. Dreiner, and U. Nierste, Correlation of $B_{s} \rightarrow \mu^{+} \mu^{-}$and $(g-2)_{\mu}$ in Minimal Supergravity, Phys. Rev. Lett. 87, 251804 (2001).

[25] J. R. Ellis, K. A. Olive, and V. C. Spanos, On the interpretation of $B_{s} \rightarrow \mu^{+} \mu^{-}$in the CMSSM, Phys. Lett. B 624, 47 (2005).

[26] S. Heinemeyer, X. Miao, S. Su, and G. Weiglein, $B^{-}$physics observables and electroweak precision data in the CMSSM, mGMSB and mAMSB, J. High Energy Phys. 08 (2008) 087.

[27] A. K. Alok and S. K. Gupta, $B_{s} \rightarrow \mu^{+} \mu^{-}$decay in the R-parity violating minimal supergravity, Eur. Phys. J. C 65, 491 (2010).

[28] F. Mahmoudi, S. Neshatpour, and J. Orloff, Supersymmetric constraints from $B_{s} \rightarrow \mu^{+} \mu^{-}$and $B \rightarrow K * \mu^{+} \mu^{-}$observables, J. High Energy Phys. 08 (2012) 092.

[29] O. Buchmueller et al., The CMSSM and NUHM1 in light of $7 \mathrm{TeV}$ LHC, $B_{s} \rightarrow \mu^{+} \mu^{-}$and XENON100 data, Eur. Phys. J. C 72, 2243 (2012).

[30] F. U. Bernlochner et al. (GAMBIT Collaboration), FlavBit: A GAMBIT module for computing flavor observables and likelihoods, Eur. Phys. J. C 77, 786 (2017).

[31] A. Djouadi et al. (MSSM Working Group), The minimal supersymmetric standard model: Group summary report, arXiv:hep-ph/9901246.

[32] S. S. AbdusSalam, The full 24-parameter MSSM exploration, AIP Conf. Proc. 1078, 297 (2009).

[33] S. S. AbdusSalam and F. Quevedo, Cold dark matter hypotheses in the MSSM, Phys. Lett. B 700, 343 (2011).

[34] S. S. AbdusSalam, Can the LHC rule out the MSSM?, Phys. Lett. B 705, 331 (2011).

[35] S. S. AbdusSalam et al., Benchmark models, planes, lines and points for future SUSY searches at the LHC, Eur. Phys. J. C 71, 1835 (2011).

[36] S. S. AbdusSalam and D. Choudhury, Higgs boson discovery versus sparticles prediction: Impact on the pMSSM's posterior samples from a Bayesian global fit, Universal J. Phys. Appl. 2, 155 (2014).

[37] S. S. AbdusSalam, LHC-7 supersymmetry search interpretation within the phenomenological MSSM, Phys. Rev. D 87, 115012 (2013).

[38] S.S. AbdusSalam, Stop-mass prediction in naturalness scenarios within MSSM-25, Int. J. Mod. Phys. A 29, 1450160 (2014).

[39] S. S. AbdusSalam and L. Velasco-Sevilla, Where to look for natural supersymmetry, Phys. Rev. D 94, 035026 (2016).
[40] M. Verzocchi, Talk at the 34th International Conference on High Energy Physics (ICHEP 2008): Philadelphia, Pennsylvania (unpublished).

[41] ALEPH Collaboration, Precision electroweak measurements on the $Z$ resonance, Phys. Rep. 427, 257 (2006).

[42] A. Abulencia et al. (CDF Collaboration), Observation of $B_{s}^{0}-\bar{B}_{s}^{0}$ Oscillations, Phys. Rev. Lett. 97, 242003 (2006).

[43] B. Aubert et al. (BABAR Collaboration), Search for the Rare Leptonic Decay $B^{-} \rightarrow \tau^{-} \bar{\nu}_{\tau}$, Phys. Rev. Lett. 95, 041804 (2005).

[44] E. Barberio et al. (Heavy Flavor Averaging Group Collaboration), Averages of $b$-hadron and $c$-hadron properties at the end of 2007, arXiv:0808.1297.

[45] E. Komatsu et al. (WMAP Collaboration), Five-Year Wilkinson Microwave Anisotropy Probe (WMAP) observations: Cosmological interpretation, Astrophys. J. Suppl. Ser. 180, 330 (2009).

[46] ATLAS Collaboration, Report No. ATLAS-CONF-2013014.

[47] CMS Collaboration, Report No. CMS-PAS-HIG-13-005.

[48] R. Aaij et al. (LHCb Collaboration), Search for the rare decays $B_{s}^{0} \rightarrow \mu^{+} \mu^{-}$and $B^{0} \rightarrow \mu^{+} \mu^{-}$, Phys. Lett. B 699, 330 (2011).

[49] R. McNabb (Muon $g$-2 Collaboration), An Improved limit on the electric dipole moment of the muon, arXiv:hep-ex/ 0407008 .

[50] E. Barberio et al. (Heavy Flavor Averaging Group (HFAG) Collaboration), Averages of $b$-hadron properties at the end of 2006, arXiv:0704.3575.

[51] K. Nakamura et al. (Particle Data Group Collaboration), Review of particle physics, J. Phys. G 37, 075021 (2010).

[52] B. C. Regan, E. D. Commins, C. J. Schmidt, and D. DeMille, New Limit on the Electron Electric Dipole Moment, Phys. Rev. Lett. 88, 071805 (2002).

[53] W. Porod, SPheno, a program for calculating supersymmetric spectra, SUSY particle decays and SUSY particle production at $e^{+} e^{-}$colliders, Comput. Phys. Commun. 153, 275 (2003).

[54] W. Porod and F. Staub, SPheno 3.1: Extensions including flavor, $C P$-phases and models beyond the MSSM, Comput. Phys. Commun. 183, 2458 (2012).

[55] B. C. Allanach, C. Balazs, G. Belanger, M. Bernhardt, F. Boudjema, D. Choudhury, K. Desch, U. Ellwanger et al., SUSY Les Houches Accord 2, Comput. Phys. Commun. 180, 8 (2009).

[56] P.Z. Skands, B. C. Allanach, H. Baer, C. Balazs, G. Belanger, F. Boudjema, A. Djouadi, R. Godbole et al., SUSY Les Houches accord: Interfacing SUSY spectrum calculators, decay packages, and event generators, J. High Energy Phys. 07 (2004) 036.

[57] G. Belanger, F. Boudjema, A. Pukhov, and A. Semenov, Dark matter direct detection rate in a generic model with micrOMEGAs 2.2, Comput. Phys. Commun. 180, 747 (2009).

[58] S. Heinemeyer, W. Hollik, D. Stockinger, A. M. Weber, and G. Weiglein, Precise prediction for M(W) in the MSSM, J. High Energy Phys. 08 (2006) 052.

[59] S. Heinemeyer, W. Hollik, A. M. Weber, and G. Weiglein, Z pole observables in the MSSM, J. High Energy Phys. 04 (2008) 039. 
[60] F. Feroz and M. P. Hobson, Multimodal nested sampling: An efficient and robust alternative to MCMC methods for astronomical data analysis, Mon. Not. R. Astron. Soc. 384 (2008) 449.

[61] F. Feroz, M. P. Hobson, and M. Bridges, MultiNest: an efficient and robust Bayesian inference tool for cosmology and particle physics, Mon. Not. R. Astron. Soc. 398 (2009) 1601.

[62] J. Skilling, Nested sampling for general Bayesian computation, Bayesian Anal. 1, 833 (2006).

[63] P. H. Chankowski and L. Slawianowska, $B_{d, s}^{0} \rightarrow \mu^{+} \mu^{-}$ decay in the MSSM, Phys. Rev. D 63, 054012 (2001).

[64] A. Crivellin, J. Rosiek, P. H. Chankowski, A. Dedes, S. Jaeger, and P. Tanedo, SUSY_FLAVOR v2: A computational tool for FCNC and $C P$-violating processes in the MSSM, Comput. Phys. Commun. 184, 1004 (2013).

[65] H. E. Logan and U. Nierste, $B_{s, d} \rightarrow \ell^{+} \ell^{-}$in a two Higgs doublet model, Nucl. Phys. B586, 39 (2000).

[66] A. Dedes, J. Rosiek, and P. Tanedo, Complete one-loop MSSM predictions for $B^{0} \rightarrow \ell^{+} \ell^{\prime-}$ at the Tevatron and LHC, Phys. Rev. D 79, 055006 (2009).

[67] G. Raven (LHCb Collaboration), Measurement of the $C P$ violation phase $\phi_{s}$ in the $B_{s}$ system at LHCb, arXiv:1212 .4140 .
[68] A. J. Buras, R. Fleischer, J. Girrbach, and R. Knegjens, Probing new physics with the $B_{s} \rightarrow \mu+\mu$ - time-dependent rate, J. High Energy Phys. 07 (2013) 77.

[69] C. Bobeth, M. Gorbahn, T. Hermann, M. Misiak, E. Stamou, and M. Steinhauser, $B_{s, d} \rightarrow l^{+} l^{-}$in the Standard Model with Reduced Theoretical Uncertainty, Phys. Rev. Lett. 112, 101801 (2014).

[70] R. Aaij et al. (LHCb Collaboration), Measurement of the $B_{s}^{0} \rightarrow \mu^{+} \mu^{-}$Branching Fraction and Effective Lifetime and Search for $B^{0} \rightarrow \mu^{+} \mu^{-}$Decays, Phys. Rev. Lett. 118, 191801 (2017).

[71] S. Chatrchyan et al. (CMS Collaboration), Measurement of the $B_{s}^{0} \vec{\mu}^{+} \mu^{-}$Branching Fraction and Search for $B^{0} \vec{\mu}^{+} \mu^{-}$ with the CMS Experiment, Phys. Rev. Lett. 111, 101804 (2013).

[72] M. Pospelov and A. Ritz, Electric dipole moments as probes of new physics, Ann. Phys. (Amsterdam) 318, 119 (2005).

[73] D. Becirevic, N. Kosnik, F. Mescia, and E. Schneider, Complementarity of the constraints on new physics from $B_{s} \rightarrow \mu^{+} \mu^{-}$and from $B \rightarrow K l^{+} l^{-}$decays, Phys. Rev. D 86, 034034 (2012). 